\title{
Le printemps érable comme choc idéologique
}

\section{Frédéric Julien}

\section{(2) OpenEdition}

\section{Journals}

\section{Édition électronique}

URL : http://journals.openedition.org/conflits/18503

DOI : $10.4000 /$ conflits. 18503

ISSN : $1777-5345$

Éditeur :

CCLS - Centre d'études sur les conflits lilberté et sécurité, L'Harmattan

\section{Édition imprimée}

Date de publication : 26 décembre 2012

Pagination : 152-159

ISBN : 978-2-336-00248-4

ISSN : 1157-996X

\section{Référence électronique}

Frédéric Julien, « Le printemps érable comme choc idéologique », Cultures \& Conflits [En ligne], 87 | Automne 2012, mis en ligne le 26 décembre 2012, consulté le 30 mars 2021. URL : http:// journals.openedition.org/conflits/18503; DOI : https://doi.org/10.4000/conflits.18503 


\title{
Le printemps érable comme choc idéologique
}

\author{
Frédéric JULIEN
}

Frédéric Julien est doctorant en science politique à l'Université d'Ottawa.

\begin{abstract}
fin de protester contre la décision du gouvernement du Québec d'aug-

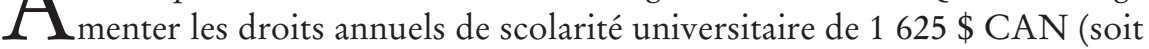
une hausse de $75 \%$ ) en l'espace de cinq ans, de nombreuses associations étudiantes de la province sont entrées en grève l'hiver dernier, à partir de la mifévrier. Jusque-là, rien d'étonnant : quel groupe organisé pourrait bien subir un tel renchérissement sans montrer les dents ? Pour autant, la perspective comptable ou corporatiste ne suffit pas à expliquer le "printemps érable ", soit la prolongation du conflit étudiant sur la saison suivante et sa mutation en une véritable crise sociale, finalement résolue par l'élection (hâtive) d'un nouveau gouvernement le 4 septembre. Les manifestations monstres, la violence, la restriction des libertés fondamentales, les concerts populaires de casseroles, tout cela peut difficilement se résumer à un enjeu pécuniaire. Pour comprendre l'intransigeance du gouvernement d'alors et la ténacité des grévistes du printemps érable, il importe donc d'examiner ce que la hausse représentait et signifiait pour chaque camp, au-delà d'une simple mesure budgétaire ou d'une majoration de facture. Cet exercice montre que les parties au conflit s'exprimaient en des termes suffisamment étrangers l'une à l'autre pour empêcher tout point de rencontre, notamment concernant leurs conceptions de l'éducation, des services publics et du processus démocratique. Par ailleurs, c'est surtout ce dernier point, la question de ce qu'est ou devrait être la démocratie, qui semble avoir motivé une portion importante de la population à se joindre au mouvement étudiant.
\end{abstract}

\section{La hausse, l'éducation et les services publics}

\section{Le discours gouvernemental}

La hausse des droits de scolarité souhaitée par le gouvernement de Jean Charest (Parti libéral du Québec, centre-droit), défait aux élections du 4 sep- 
tembre, relevait d'un plan de financement visant à fournir « [...] de nouveaux moyens aux universités, piliers importants de l'économie du savoir ${ }^{1}$ ». Pour le Premier ministre et son équipe, l'intérêt d'investir davantage dans la « formation universitaire » tenait en effet à la « rentabilité publique » de l'exercice, c'est-à-dire au gain obtenu par la société « [...] en raison des avantages retirés sur le plan de la compétitivité accrue et de la participation active aux secteurs à haute valeur ajoutée 2 ». Or, pour être compétitif, il faut pouvoir disposer de ressources au moins équivalentes à celles de ses concurrents. D’après le gouvernement Charest, ce n'était pas le cas des universités québécoises en raison de leurs déficits budgétaires accumulés et de revenus de fonctionnement inférieurs à ceux de leurs rivales du reste du Canada, lesquelles furent retenues comme unique base comparative ${ }^{3}$. Le gouvernement Charest soulignait en outre la « rentabilité privée » de la formation universitaire, l'obtention d'un baccalauréat (l'équivalent québécois d'une licence) permettant d'espérer « [...] un gain salarial additionnel de près de 600000 [CAN] sur la durée de vie active d'une personne ${ }^{4}$ » par rapport à un diplôme d'études secondaires. Les « taux de rendement privé très élevés » sur l'investissement dans une formation universitaire seraient même supérieurs aux «taux de rendement public » dont jouit l'ensemble de la société ${ }^{5}$. De ce point de vue, il était raisonnable d'augmenter le coût d'une formation universitaire de quelques milliers de dollars afin de bonifier les budgets des universités. D’ailleurs, comme le notait aussi le gouvernement Charest, c'est précisément le chemin qu'ont emprunté les compétiteurs de la Belle Province dans le reste du Canada ${ }^{6}$.

Ainsi, c'est une représentation de l'éducation comme capital humain qui semble avoir nourri la ferme volonté du gouvernement Charest de relever les droits de scolarité : «[...] l'éducation est importante parce qu'elle permet aux travailleurs d'être plus productifs et, de la sorte, d'être capables de gagner un salaire plus élevé 7 ». Le développement économique du Québec fut en tout cas la seule justification donnée par le gouvernement pour son plan de financement des universités et le retour sur investissement dans une formation universitaire fut l'unique raison offerte pour motiver l'accroissement de la contribution étudiante aux budgets des universités. Le modèle éducatif du capital humain paraît avoir été d'autant plus déterminant pour le gouvernement que la hausse s'inscrivait dans un plan global de réforme du financement des ser-

1. Ministère des Finances, Un plan de financement des universités équitable et équilibré : pour donner an Québec les moyens de ses ambitions, Québec, Gouvernement du Québec, mars 2011, p. 3.

2. Ibid., p. 26.

3. Ministère de l'Éducation, du Loisir et du Sport, L'avenir des universités et leur contribution au développement du Québec, Québec, Gouvernement du Québec, décembre 2010, pp. 2125.

4. Ibid., p. 61.

5. Ibid.

6. Ministère des Finances, op. cit., p. 24.

7. Robeyns I., "Trois modèles "éducatifs" : droit, potentialité et capital humain ", Les ateliers de l'éthique, vol. 6, n 1, 2011 [2006], p. 20. 
vices publics, dont l'objectif était de provoquer une "révolution culturelle » basée sur le principe de «l'utilisateur-payeur », à qui l'on demandait de fournir sa « juste part » 8 . Concrètement, le mariage de l'éducation comme capital humain et de la juste part signifie que sur les 850 millions de dollars additionnels dont le gouvernement voulait doter le réseau universitaire québécois, $31,2 \%$ devaient provenir de la hausse des droits de scolarité, alors que la contribution étudiante actuelle au financement global des universités est plutôt de l'ordre de $12,7 \%$ '.

\section{Le discours des grévistes}

Selon le mouvement étudiant, des droits de scolarité substantiels réduisent l'accessibilité de l'éducation universitaire pour les personnes moins fortunées et la classe moyenne, une injustice qui n'est qu'imparfaitement compensée par les programmes d'aide financière aux études et qui serait magnifiée par une hausse des droits. Le combat des grévistes a cependant dépassé la seule question de l'accès aux études. L'aile la plus militante du mouvement étudiant, regroupée au sein de la Coalition large de l'Association pour une solidarité syndicale étudiante (CLASSE, désormais « en dormance »), revendique la gratuité de l'éducation universitaire, mais aussi de tous les services publics. Même pour l'aile plus modérée, qui se limite à demander le gel des droits de scolarité, refuser la hausse, c'était aussi refuser la « [...] pensée dominante qui répète inlassablement des mantras prônant le désengagement de l'État, la privatisation des services publics, le laisser-faire économique et le tout au marché [...], alors même que des droits sociaux et économiques fondamentaux sont en cause $10 »$. Cette inscription du refus de la hausse dans une lutte plus large contre la tentation néolibérale du gouvernement Charest, animé par un désir de « réingénierie » managérialiste de l'État depuis son arrivée au pouvoir ${ }^{11}$, paraît avoir joué un rôle clef dans la mobilisation étudiante. D’une part, prônant un "syndicalisme de combat », la CLASSE a attiré à elle des dizaines de milliers de membres supplémentaires à mesure que s'enlisait le conflit avec le gouvernement ${ }^{12}$. Alors que la scène politique étudiante québécoise était dominée par le «syndicalisme de concertation » depuis les années 1990, la CLASSE a su imposer sa démarche, une évolution à mettre en parallèle avec l'émergence de la galaxie altermondialiste dans les années 2000 13. D’autre

8. La Presse canadienne, «Bachand défend le principe de l'utilisateur-payeur », La Presse, vol. 128, n 124, 12 mars 2012, p. A10.

9. Ministère des Finances, op. cit., p. 17.

10. Alliance sociale, «Un autre Québec est possible », Le Devoir, vol. 101, n 251, 5 novembre 2010 , p. A9.

11. Fortier I., "La “réingénierie de l’État”, réforme québécoise inspirée du managérialisme ", Revue française d'administration publique, $\mathrm{n}^{\circ} 136,2010$, pp. 803-820.

12. Duchaine G., "La CLASSE a le vent dans les voiles », La Presse, vol. 128, n 169,5 et 6 mai 2012, p. A8.

13. Nagy P. et Petitclerc M., «La grève est étudiante, la lutte est populaire », Revue du MAUSS permanente, 5 juin 2012 (http://journaldumauss.net/spip.php?article902, consulté le 20 août 2012). 
part, l'élargissement de la lutte a permis aux grévistes d'obtenir le soutien des grandes centrales syndicales ${ }^{14}$ et d'autres groupes de la société civile. Au cœur du combat étudiant contre ce qui est perçu comme une économicisation et une atomisation de la société, il y a la vision de «l'éducation comme potentialité », qui « [...] invite à prendre en compte toutes les sources d'inégalités qui réduisent le champ des possibles des individus $15 »$. De fait, pour la CLASSE, éduquer c'est mettre « $[\ldots]$ à la disposition de toutes et tous les ressources nécessaires au plein développement de leurs capacités 16 »dans un but d'émancipation individuelle et collective. En outre, l'éducation est un « apprentissage de l'humanité » plutôt qu'une "branche de l'économie » ou un "service de formation à court terme 17 ». Dans cette perspective, les droits de scolarité sont une des nombreuses « [...] entraves à la pleine réalisation de notre humanité 18 » et le gel des droits, une mesure minimale. La CLASSE milite plutôt en faveur de la gratuité, plus juste, parce que « [1]a gratuité, c'est payer ensemble ce que l'on possède ensemble 19 ».

\section{La hausse et le processus démocratique}

L'appui populaire au mouvement étudiant lors du printemps érable fut surtout celui du peuple de gauche du Québec 20 et, nous l'avons vu, l'opposition à la hausse a servi de point de rencontre à celles et ceux qui contestaient l'ensemble des politiques d'inspiration néolibérale du gouvernement Charest. Si le peuple de gauche du Québec est sorti dans la rue, ce n'est cependant pas simplement pour protester contre ce qu'il percevait comme une politique néolibérale de trop. La manière de procéder du gouvernement Charest, les moyens qu'il a retenus pour imposer cette politique ont peut-être même pesé plus lourd dans la balance que la hausse des droits elle-même. De fait, c'est suite à la loi spéciale du 18 mai qu'a fait voter le Premier ministre afin de suspendre les cours affectés par la grève étudiante et d'encadrer strictement les rassemblements publics que l'appui populaire au mouvement étudiant s'est le plus clairement fait sentir. Chaque soir à $20 \mathrm{~h}$, deux semaines durant, des mil-

14. Castonguay A., « Le poids des syndicats », L'Actualité.com, 5 juin 2012 (http://www.lactualite.com/societe/le-poids-des-syndicats?page $=0,0$, consulté le 20 août 2012).

15. Robeyns I., op. cit., p. 25.

16. CLASSE, "Ensemble, nous sommes capables de beaucoup" ", Le Devoir, vol. 103, n 155, 12 juillet 2012, p. A7.

17. Ibid.

18. Ibid.

19. Ibid.

20. Dans un sondage effectué à la mi-juin, les personnes ayant exprimé une intention de vote pour un des deux principaux partis politique québécois à la droite du centre appuyaient massivement la hausse des droits de scolarité. À l'opposé, celles ayant exprimé une intention de vote pour un des deux principaux partis à gauche du centre soutenaient fortement le combat des associations étudiantes. Une analyse sommaire des propos tenus par les journalistes d'opinion confirme ce clivage gauche-droite au-delà des seules lignes partisanes et de la traditionnelle opposition québécoise entre indépendantistes et fédéralistes (Léger Marketing, La politique provinciale et fédérale an Québec, rapport de sondage, 16 juin 2012, p. 13 [http://www.legermarketing.com/admin/upload/publi_pdf/Sondage\%20politique\%20provinciale_Le\%20Devoir_15\%20juin\%202012_final.pdf, consulté le 20 août 2012]). 
liers de personnes de toutes générations et des quatre coins du Québec ont usé leurs casseroles et leurs cuillères dans un grand tintamarre citoyen afin de signifier leur ras-le-bol. Quatre jours après l'entrée en vigueur de la loi, des dizaines de milliers de personnes ont pris d'assaut les rues du centre-ville de Montréal dans le but de manifester leur colère devant le coup de force gouvernemental. Dans les sondages d'opinion, l'appui au mouvement étudiant, alors en baisse, est remonté subitement et fortement (tout en demeurant minoritaire) ${ }^{21}$. Si le Premier ministre en est venu à « [...] porte[r] atteinte à des libertés fondamentales garanties par la Charte des droits et libertés de la personne $22 »-$ dixit l'organisme officiellement chargé de veiller au respect desdites libertés -, c'est apparemment pour défendre la démocratie et l'État de droit. Selon lui, celles et ceux qui ont pris la rue en invoquant la hausse des droits de scolarité visaient en fait à « [...] contester l'ordre établi et ses institutions démocratiques, incluant l'Assemblée nationale et aussi nos tribunaux 23 ». D'ailleurs, pour Jean Charest, la rue et la démocratie semblaient s'opposer : «Est-ce qu'au Québec, c'est la rue qui gouverne ? [...] Ou est-ce que ça va être [...] la démocratie 24 », demandait-il lors de la récente campagne électorale. Bien sûr, il convenait qu'en démocratie, manifester sa désapprobation quant aux politiques gouvernementales est un droit. Seulement, déclaraitil suite à l'interruption définitive des négociations avec les associations étudiantes, si les contestataires « $[\ldots]$ avaient des messages à passer, $[\ldots]$ les messages ont été passés » et poursuivre la contestation serait «[...] nuire aux Québécois ». Pour le reste, le gouvernement avait fait ses choix et les élections à venir seraient « [...] le moment pour les gens d'exprimer leur désaccord, démocratiquement 25 ». Même Line Beauchamp, la ministre de l'Éducation qui a démissionné peu de temps avant l'adoption de la loi spéciale et qui a refusé depuis de dire si elle soutenait cette initiative, n'en pensait apparemment pas moins : "Les élus du peuple n'ont jamais tort, ils ont toujours raison. Le plus important est de [les] respecter 26 ». Avec le politologue Marc Chevrier, on peut reconnaître dans ces propos une vision libérale classique de la démocratie représentative :

21. Ibid., p. 12.

22. Commission des droits de la personne et des droits de la jeunesse, Loi spéciale visant à assurer le retour en classe des étudiants : des dispositions de la Loi portent atteinte à la Charte des droits et libertés de la personne, selon la Commission des droits de la personne et des droits de la jeunesse, communiqué de presse, 19 juillet 2012 (http://communiques.gouv.qc.ca/ gouvqc/communiques/GPQF/Juillet2012/19/c9413.html, consulté le 20 août 2012).

23. Dutrisac R., « La voix de la majorité silencieuse », Le Devoir, vol. 103, n 173, 2 août 2012, p. A8.

24. Boisvert L., "Jean Charest interpelle la majorité silencieuse », Radio-Canada.ca, 2 août 2012 (http://www.radio-canada.ca/sujet/elections-quebec-2012/2012/08/01/009-charest-debutcampagne.shtml, consulté le 20 août 2012).

25. "Jean Charest renouvelle son appel au calme », Radio-Canada.ca, 1er juin 2012 (www.radiocanada.ca/nouvelles/Politique/2012/06/01/001-charest-vendredi-reaction-criseetudiante.shtml, consulté le 20 août 2012).

26. Corbeil M., « Beauchamp refuse de dire si elle appuie la loi 78 », Le Soleil, vol. 116, n 215, 4 août 2012, p. 7. 
«Bien sûr, on peut contester les décisions et manifester, mais la véritable initiative vient du gouvernement. Et quand on n'est pas content, on change de gouvernement aux prochaines élections. [...] Le peuple en lui-même n'est pas une entité [...]. Ce sont les individus titulaires de droits qui comptent. La société, elle, demeure très policée et elle est définie par les droits contractuels de tout un chacun. Dans ce portrait, les lieux publics sont faits pour la circulation $[\ldots]$ et toute atteinte à cette circulation devient une atteinte aux droits contractuels. 27 »

De fait, contrairement à l'usage coutumier, le gouvernement Charest a préféré parler de «boycott » des cours plutôt que de grève pour désigner le moyen de pression choisi par le mouvement étudiant. Dans le discours gouvernemental, les piquets de grève ont donc cessé d'être des actes de solidarité visant à faire respecter un choix collectif contraignant, pour devenir des actes d' « intimidation » à l'égard des individus qu'on empêchait de suivre une formation qu'ils avaient payée et à laquelle ils avaient le «droit le plus strict » 28 . En niant ainsi toute légitimité démocratique au mouvement étudiant, le gouvernement Charest a d'ailleurs encouragé la juridicisation du conflit, plusieurs personnes ayant demandé (et obtenu) une injonction légale pour forcer la tenue des cours (sans grand effet toutefois sur les piquets de grève) ${ }^{29}$. De son côté, la CLASSE raille une démocratie représentative dont «[...] on se demande bien qui elle représente [et qui] ne se vit qu'une fois tous les quatre ans 30 ». Conséquemment, « [l]e processus électoral n’est pas le moyen qu'utilise [la] CLASSE pour arriver à ses fins 31 ». S'efforçant de pratiquer la démocratie directe, la CLASSE rêve « [...] d'une prise en charge permanente de la politique par la population, à la base, comme premier lieu de la légitimité politique 32 ». L'aile plus modérée du mouvement étudiant a plutôt choisi d'investir le jeu électoral en encourageant les jeunes, grands abstentionnistes, à se présenter aux urnes pour battre le gouvernement Charest aux élections du 4 septembre dernier. Une des figures de proue de cette partie du mouvement a d'ailleurs fait le choix d'entrer en politique professionnelle en rejoignant les rangs du parti d'opposition d'alors - le Parti québécois, centre-gauche, aujourd'hui au gouvernement -, mais tout en précisant que sa décision était «surtout » motivée par la possibilité « [...] de bâtir une démocratie plus parti-

27. Baillargeon S., « Monarcho-libéraux contre républicains », Le Devoir, vol. 103, n 123, 2 et 3 juin 2012, p. A12.

28. Richer J. et Robillard A., "L’intimidation doit cesser, dit Charest », Le Nouvelliste, vol. 92, $\mathrm{n}^{\circ} 140,12$ avril 2012 , p. 3 .

29. Saint-Arnaud P., «Les étudiants se heurtent aux injonctions », Le Droit, vol. 100, $\mathrm{n}^{\circ} 15,13$ avril 2012, p. 20.

30. CLASSE, " "Ensemble, nous sommes capables de beaucoup" ", op. cit.

31. CLASSE, "La CLASSE appelle à la poursuite de la grève et de la mobilisation populaire », communiqué de presse, 12 août 2012 (www.bloquonslahausse.com/2012/08/la-classeappelle-a-la-poursuite-de-la-greve-et-de-la-mobilisation-populaire/, consulté le 20 août 2012).

32. CLASSE, " "Ensemble, nous sommes capables de beaucoup” ", op. cit. 
cipative 33 ». Le peuple n'a pas de porte-parole, mais en prenant la rue et en faisant résonner ses casseroles, une partie importante de la population a au minimum signifié son rejet du fétichisme électoral et exigé une démocratie "plus participative ». Par ailleurs, dans un sondage publié suite à l'adoption de la loi spéciale, alors que $43 \%$ des personnes interrogées ont affirmé être "davantage favorable à la position des étudiants 34 » dans le conflit étudiant, $53 \%$ d'entre elles considéraient que la loi spéciale « va beaucoup trop loin (est beaucoup trop sévère) 35 ». De surcroît, 76 \% des individus sondés ont dit souhaiter que les négociations reprennent entre le gouvernement Charest et les associations étudiantes ${ }^{36}$. À la loi spéciale et à l'intransigeance gouvernementale, les « casserolistes » auraient assurément préféré des états généraux sur l'éducation, qui furent de fait réclamés par les associations étudiantes nationales et d'autres groupes de la société civile (et accordés par le nouveau gouvernement du Parti québécois). Une telle consultation sur le devenir des universités et collèges, notamment leur financement, correspond en principe à un exercice de «démocratie délibérative 37 », où « [...] la norme n'est pleinement légitime que si elle est fondée sur des raisons publiques résultant d'un processus de délibération inclusif et équitable, auquel tous les citoyens peuvent participer et dans lequel ils sont conduits à coopérer librement 38 ». Cette perspective délibérative s'oppose évidemment « [...] aux conceptions libérales construisant de façon cumulative l'intérêt général comme simple addition ou négociation entre les intérêts particuliers 39 \%.

\section{Conclusion}

La mise en parallèle des discours du gouvernement Charest et des grévistes du printemps érable montre l'indéniable fossé idéologique qui les séparait : le débat central n'a pas porté sur la somme que devrait verser celui ou celle qui entreprend des études - le niveau de la « juste part »-, mais sur la légitimité même de l'application du principe de l'utilisateur-payeur à l'éducation universitaire. Le gouvernement s'est montré d'autant plus intransigeant que la hausse des droits de scolarité s'inscrivait dans un plus large projet de

33. Bureau-Blouin L., " "Faire une différence" pour tous les Québécois ", Le Devoir, vol. 103, $\mathrm{n}^{\circ} 166,25$ juillet 2012, p. A7.

34. Léger Marketing, La loi qui divise, rapport de sondage, 21 mai 2012, p. 5 (www.legermarketing.com/admin/upload/publi_pdf/Sondage_loi_speciale-21mai.pdf, consulté le 20 août 2012).

35. Ibid., p. 7.

36. Ibid., p. 10.

37. Les concepts de démocratie participative et de démocratie délibérative sont différents. «En dépit de nuances importantes, l'intention première est cependant la même : il s'agit de compléter l'arsenal institutionnel de la démocratie représentative par des lieux où le déploiement [d'une] délibération démocratique élargie est possible, où [la] participation du plus grand nombre à [la] discussion des choix collectifs est encouragée. " (Blondiaux L., "La démocratie participative, sous conditions et malgré tout : un plaidoyer paradoxal en faveur de l'innovation démocratique ", Mouvements, ${ }^{\circ}$ 50, juin-août 2007, pp. 120-121).

38. Blondiaux L., Sintomer Y., "L’impératif délibératif », Rue Descartes, n 63, 2009, p. 28.

39. Ibid., pp. 28-29. 
« révolution culturelle » et que son discours sur la « juste part » et la défense de la démocratie libérale résonnait dans la population, à tel point qu'il y a vu une fenêtre électorale 40 et qu'il a déclenché un scrutin hâtif - finalement perdu, mais de peu. Bien que le coût financier du printemps érable ait probablement dépassé les sommes que la première année de hausse devait rapporter aux universités ${ }^{41}$, le Premier ministre a maintenu une ligne dure. De son côté, le mouvement étudiant s'est révélé d'autant plus tenace que la lutte à la hausse s'est insérée dans une critique d'ensemble de l'œuvre (néolibérale) du gouvernement Charest, un discours mobilisateur pour les grévistes et dont le pouvoir d'interpellation a dépassé le seul milieu étudiant. Les grévistes ont fait du gel des droits une condition minimale à toute entente avec le gouvernement, au point de compromettre leur trimestre et de proposer au gouvernement d'échanger certains privilèges fiscaux pour études contre une réduction équivalente de la hausse des droits, un jeu de vases communicants qui n'aurait rien apporté aux grévistes d'un point de vue purement comptable ${ }^{42}$. Si l'on peut certes considérer qu'un discours sonne plus juste que l'autre, on aurait toutefois tort de les considérer comme de simples armes rhétoriques, d'une part, d'un gouvernement cherchant à boucler un budget ou à assurer sa réélection et, d'autre part, d'un groupe de pression défendant les intérêts pécuniaires de ses membres. Malgré les coûts sociaux et financiers occasionnés par le printemps érable, la population québécoise n'en a pas moins eu droit à un véritable exercice politique. De quoi être heureux d'un printemps.

40. Léger J.-M., « La zone payante », Le Journal de Montréal, vol. 49, n 5, 19 juin 2012, p. 23.

41. Breton P., «La grève éclipse la hausse », La Presse, vol. 128, n 160, 25 avril 2012, p. A5.

42. "Offres étudiantes et gouvernementales ", Radio-Canada.ca, 1er juin 2012 (www.radiocanada.ca/nouvelles/Politique/2012/06/01/002-offres-etudiants-gouvernement.shtml, consulté le 20 août 2012). 